\title{
Map-Based Navigation in a Graphical MOO
}

\author{
Wendy A. Schafer, Doug A. Bowman, and John M. Carroll \\ Center for Human-Computer Interaction \\ Department of Computer Science \\ Virginia Tech \\ Blacksburg, VA 24061 USA \\ Phone +1 5402317524 \\ Fax +15402316075 \\ \{wschafer, bowman, carroll $\} @$ vt.edu
}

\begin{abstract}
Traditional MUDs and MOOs lack support for global awareness and simple navigation. These problems can be addressed by the introduction of a map-based navigation tool. In this paper we report on the design and evaluation of such a tool for MOOsburg, a graphical 2D MOO based on the town of Blacksburg, Virginia. The tool supports exploration and place-based tasks in the MOO. It also allows navigation of a large-scale map and encourages users to develop survey knowledge of the town. An evaluation revealed some initial usability problems with our prototype and suggested new design ideas that may better support users. Using these results, the lessons learned about map-based navigation are presented.
\end{abstract}

\section{Keywords}

map-based navigation, collaborative virtual environments, awareness, fisheye views, focus+context techniques

\section{INTRODUCTION}

Traditional MUDs and MOOs provide limited interaction and navigation. In a MUD (multi-user domain) or MOO (MUD object-oriented), users interact with one another and with the objects in the environment through simple textual commands. The interface is a scrolling sequence of commands and descriptions that pertain to the activities occurring in a particular room of the environment. Actions such as a user entering/leaving the room, speaking in the room, and picking up an object are displayed in the sequence. Movement through the world is accomplished with other textual commands such as 'go north'. Users navigate from room to adjacent room using their knowledge about the layout of the environment. This knowledge is acquired through repeated interaction with the environment, so that frequent users have a good mental model of the environment and new users have no knowledge of the spatial layout. 
It is particularly difficult for these interfaces to provide awareness information. The scrolling sequence of activity provides the user with awareness about a particular room, but does not provide any information about the other users or activities occurring in the environment. Users only learn about activities in a room if they enter that room by chance. This prevents users from finding interesting objects and activities in the environment and it limits the opportunities for collaboration between users.

Traditional interfaces also limit a user's navigation of the environment. With most of the movement commands pertaining to an adjacent room, navigation through the environment occurs through multiple, repeated commands. This step-by-step process is not only tedious, but it also requires the user to recall the spatial layout in order to navigate between distant rooms. The layout is based on a directed graph such that going north and then east is not necessarily the same as going east and then north. Users must develop a mental model of this layout through repeated use. In gaming environments, this learning of the spatial layout may be part of the fun. But in MOOs primarily used for interacting with objects and other users, it may distract the user from other more relevant tasks.

Map-based navigation provides one solution to the problems of traditional MUDs and MOOs. In map-based navigation, the text-based interface is combined with a graphical map of the environment. In this way, users do not have to develop a mental model of the spatial layout nor remember it. Also, the map can provide direct access to rooms through a clickable interface, eliminating the tedious movements. In addition, global awareness information can be displayed on the map. The map provides a graphical overview of the environment, which can be designed to provide awareness cues visually. For example, the map can indicate rooms with many objects, rooms with many users, or rooms that are visited most often. This should guide users to find interesting activities and encourage collaboration between users.

This paper addresses some of the design challenges introduced by map-based navigation. It describes the design of a mapbased navigation tool for MOOsburg, a graphical MOO based on the town of Blacksburg, Virginia. This design supports the navigation of large-scale maps with detailed information through fisheye views and a continuous zoom feature. The design also supports a user's mental representation of Blacksburg and it encourages corrections in this representation. Lastly, the design addresses issues related to designing maps for diverse user groups.

\section{RELATED WORK}

Thorndyke and Hayes-Roth describe three types of spatial knowledge (Thorndyke and Hayes-Roth 1982). Landmark knowledge is a familiarity with the visual attributes of an environment; Route knowledge is a familiarity with paths through an environment; and survey knowledge is a familiarity with an overview layout of an environment, similar to a map. People 
who develop a cognitive map of an environment through first-hand active navigation are likely to have an orientationdependent map representation. Users of MOOsburg will be familiar with traveling through town as opposed to looking at a north-up map of town. This implies that users will have greater landmark knowledge and route knowledge than survey knowledge.

Lynch proposed that people use paths (e.g. walkways and passages), edges (e.g. walls and fences), landmarks (e.g. church spire), nodes (objects with similar characteristics), and districts (distinct sections) to build their cognitive maps (Lynch 1960). By placing these objects on a map, the map will better align with the users' conception of a space and better support the formation of spatial knowledge. For example, by including major landmarks in Blacksburg on our map, users will be able to make a connection between landmarks in their mental representation and the landmarks on the map interface.

Map-based navigation has been used in virtual environments (Bowman, Wineman, Hodges, and Allison 1999). Working with a north-up map, users can drag an icon of themselves from one location to another resulting in animated travel through the environment. Maps also support search tasks in virtual environments because they provide the user with survey knowledge that may not be learned through navigating the environment (Darken and Sibert 1996). Map-based navigation of a MOO differs in that the map is used to navigate directly and is not just an aid for navigation.

Fisheye views use focus+context techniques to present the details needed for local interaction, but also include a compressed view of the overall structure (Furnas 1982; Lamping, Rao, and Pirolli 1995). Sarkar and Brown present a way to implement fisheye views for graphs using geometric transformations (Sarkar and Brown 1992). In their work, they suggest a specialized transformation for maps to make the fisheye view more natural. This transformation is different from others in that it not only makes items in the focus larger but it projects the map onto a hemisphere. We explore several projections in our map-based navigation tool.

\section{MAP-BASED NAVIGATION TOOL}

\section{MOOsburg}

MOOsburg explores the use of a graphical MOO as a community application (Carroll, Rosson, Isenhour, Ganoe, Dunlap, Fogarty, Schafer, and Van Metre 2001). Most MUDs and MOOs are based on fictional places and are intended for almost any user group. MOOsburg, on the other hand, is based on a real place, the town of Blacksburg, Virginia, and its target user group is the town populace.

These unique characteristics make map-based navigation a particularly good interface solution for MOOsburg. Because MOOsburg is based on an actual place, maps already exist for that place and we can reuse these maps in the interface. In 
addition, we can tailor the design to community members and make use of community knowledge such as major town landmarks.

The structure of MOOsburg is also different than traditional MOOs. Instead of a network of rooms based on a directed graph, MOOsburg consists of spaces and landmarks, which correspond to points in a Cartesian space. Spaces are places in town that contain other places. Landmarks are visitable places in the MOO. A user either navigates to a landmark, or, if the landmark is itself a space with a substructure, the user can go into the landmark. Buildings are typical spaces and rooms are typical landmarks within these spaces. The map needs to support this hierarchy, as users will explore both spaces and landmarks. Figure 1 shows the current MOOsburg interface including the map tool prototype.

The goal of MOOsburg is support community development by providing access to local information and to new collaboration activities. The project incorporates end-user programming to encourage relevant content and community involvement. In particular, users can add new landmarks and spaces to the MOO as well as develop new objects that provide collaboration opportunities.

\section{Insert figure 1 here}

\section{Usage scenarios}

Based on this description, the usage scenarios for MOOsburg are fairly different from those of traditional MUDs and MOOs. They focus on users finding information and collaborating with other users, whereas traditional MOO scenarios often focus on users navigating the environment and learning the spatial layout. This difference reflects the need for a different interface. In particular, the MOOsburg's interface should support efficient navigation and provide awareness information. Map-based navigation fulfills these requirements.

We anticipate three primary types of tasks in MOOsburg - exploration, goal-based tasks, and developer tasks. Many users will simply want to explore the MOO. This is the expected scenario for novice users and users just looking for interesting activities. For example, a new user will investigate the different spaces in MOOsburg and visit many landmarks. This is the exploration scenario.

Secondly, users may log on to MOOsburg with a particular task in mind. This task may be related to a specific place, such as visiting the Virtual Science Fair at the Blacksburg Middle School, or it may be more general, such as learning the locations of all the coffee shops in town. In the Virtual Science Fair example, a user would work with the map to locate the school and then enter the school space to find the science fair. In the coffee shop example, the user would execute a search function that would cause the map to highlight all the places to get coffee. Both examples are goal-based scenarios. 
Lastly, the map interface needs to support end-user programming tasks. In particular, users need to be able to add spaces and landmarks to the map as they add content to the MOO.

Our current prototype focuses on exploration tasks and place specific, goal-based tasks. A search feature will be investigated in the next version of the prototype to support other goal-based tasks. Also, providing support for end-user programming was not a priority in the prototype, as MOOsburg was still under development itself at the time.

\section{Map prototype}

Our prototype tool provides map-based navigation in MOOsburg and allows us to investigate the usability of different map features. The prototype provides an accurate representation of the town through the use of digital data acquired from town authorities.

The format of this data, ESRI shapefiles, enables GIS-style display and interaction techniques. Stored in the form of layers, the data allows either a single layer or multiple layers to be displayed. For example, we can display the road layer at certain zoom levels and both the building and road layers for more zoomed-in views. Each layer of data consists of a set of vector coordinates for the geometry of the objects in that layer. In this way, we can apply mathematical functions (Arc Tan, Hyperbolic, Parabolic $\mathrm{x}^{2}$, and Parabolic $\mathrm{x}^{1.5}$ ) to the coordinates to produce different projections. In the map prototype, the different fisheye views correspond to different projections (see figure 3). The format of the data also allows a continuous zoom feature because it is vector-based.

The map supports three main types of interaction - clicking, dragging, and zooming. When users click on the interface, the map is redrawn with the location of the click placed in the centre of the map. Dragging the map produces a panning motion, revealing parts of the map not previously visible. Lastly, users can change the zoom factor of the map continuously through a slider widget. Figures 2 and 3 illustrate these map interactions.

Insert figure 2 here

Insert figure 3 here

The map in MOOsburg needs to address the transformation from a mental representation of Blacksburg to an actual map. People need to recognize the map as the town of Blacksburg and be able to relate to it. We hypothesized that including major 
landmarks in town on the map would allow users to make a connection between places in their mental representation and major town landmarks we provide on the map.

We can also support route knowledge, by including the roads and pathways of Blacksburg. However, a map that can be rotated might better support the users' orientation-dependent spatial knowledge (Darken and Cevik 1999).

Lastly, as the map displays survey knowledge of the town this may cause users to correct any inconsistencies with their mental representation. In working with the map, users will gain a better understanding of the layout of the town. This knowledge will be helpful as users continue to interact with the map and it may cause users to develop a more accurate cognitive map of Blacksburg.

\section{FORMATIVE EVALUATION}

Following the implementation of the map prototype, we conducted a formative evaluation. This evaluation revealed some initial usability problems with our prototype and introduced some new design ideas that may better support users.

\section{Subjects}

The evaluation of the map prototype involved subjects from three different user groups - middle school students, college students, and seniors. MOOsburg is a community application and so it is important to have a variety of community members involved in its evaluation. We wanted to avoid an interface design that is useful for college students and yet causes problems for middle school students and seniors. In particular, we were uncertain about how well the middle school students would be able to work with a map of the town, since at this age map skills and spatial knowledge may not be well developed. Also, we expected the evaluation to reveal differences in the user groups and indicate if different user groups would benefit from different interface designs.

We met with four users from each user group for a total of twelve subjects. Within each group, two of the users were female and the other two were male. The average ages of the middle school students, college students, and seniors were fourteen, twenty-three, and seventy-seven, respectively. All the users indicated that they use computers frequently to do a variety of tasks. Most of the college students indicated that they use map programs over the Internet once a month and that they consult atlases and other paper maps less frequently. None of the seniors or eighth graders had used Internet map programs previously, but both groups indicated monthly use of atlases and paper maps.

\section{Method}

Each user followed a similar four-step procedure that lasted about 20 minutes. The evaluation was intended to be think-aloud and users were asked to comment on their thoughts while performing the tasks. 
After filling out a background questionnaire, the users answered a series of questions related to flat map paper mockups. The first mockup was an unlabeled map of major roads in Blacksburg and we asked the user if he/she recognized the place depicted in the map. This was to investigate how familiar users were with the layout of Blacksburg and whether they could recognize it from just a sketch of the major roads.

We also looked at the users' ability to work with undetailed, overview maps of the town and whether they could understand one abstraction better than another. Ideally, users will be able to navigate to any place in MOOsburg if given an overview map of the town. We investigated what details need to be provided on this overview map to support navigation. To do this, we examined how successfully users pointed out places in town with three different maps - a map of major roads without labels, a map of major roads with labels, and a map with a set of possible major town landmarks labeled, in addition to major roads without labels.

During this line of questioning, we noted road names and town landmarks the users mentioned. This allowed us to learn about the users' perceptions of the major roads and landmarks in town, where repeated use of certain roads and landmarks indicates their significance. We also observed how the users worked with maps. In particular, actions such as drawing on the map and rotating the map were noted. These actions would indicate that features such as editing or rotating capabilities might improve the usability of the map interface.

In the second step, users worked with the map prototype to complete two tasks. The first task asked users to find and centre their home with no further instructions on how to work with the map. This allowed us to observe how easy it is to learn how to interact with the map and how intuitive the map interactions are. In the second task, we described for the users the three interaction techniques the prototype supports and asked the user to centre the downtown area. This gave the users an opportunity to experiment with the map further and try out all the features, while we again observed the intuitiveness of the interactions and the map's ease of use.

In the third section of the evaluation, users were asked to point out four different places using four different fisheye view paper mockups. Each view showed the roads and buildings in one section of town. Observations were made about the users' initial reactions to the views in addition to how the users worked with the maps.

Lastly, the users worked with the three fisheye views of the prototype. The users were asked to navigate to the places they pointed out in the previous step (this tested the goal-based navigation usage scenario). Two trials were run for each place where each involved a different view and different starting position. The first task compared two different fisheye views. In the second and third tasks the other two fisheye views were compared with the flat map view. Users were allowed to use the 
zoom feature for the second task but not the third. We collected the users' comments while working with the different views, their view preference after completing the tasks, and the time taken to complete the tasks. This allowed us to examine the usability advantages fisheye views may have over the flat map view.

\section{RESULTS}

\section{Section 1: Paper prototypes of a flat map}

We first observed the users working with different paper mockups of the town. When they were asked to identify Blacksburg from an overview map of major roads, only two of the eighth graders recognized the town, while three college students and all of the seniors answered correctly. After we informed the users that it was Blacksburg, some of the seniors and college students could point out their current location. Yet, none of the eighth graders could point out an approximate location for their current location, the Blacksburg Middle School. The school lies on the main road running through town, yet all of the eighth graders struggled to work with the map.

When major road names were added, it was apparent that almost all of the users were familiar with the location of the grocery stores. Each senior pointed out two grocery stores when asked to point out where they shop. All of the college students were familiar with each grocery store's location. Three out of the four eighth graders knew what road their grocery store was located on, but only two of them could point it out.

Next, the users were presented with a similar map with landmarks. Some of the college students were not familiar with all of the landmarks displayed, while all the seniors and the eighth graders indicated that they recognized the landmarks. For example, many of the college students could not identify with the different public schools on the map. All of the college students indicated that the labeled roads map was much more helpful than the landmark map (one user even requested to use the map with road labels to answer the question!). Similarly, many of the seniors indicated that the roads names were more helpful.

During this section of the test, five of the twelve users were observed drawing on the paper mockups and at least one user from each group rotated a paper mockup.

\section{Section 2: Interacting with a flat view of the prototype}

Observing users experimenting with the map prototype revealed some key usability problems with the prototype. Most importantly, our observations showed that the clicking interaction technique is not intuitive. During first use, all of the seniors were confused by the tool's response to a click or they commented that clicking was confusing. Similarly, most of the 
college students used clicking just to move the map and did not realize that the map was re-centering where they had clicked. Two of the eighth graders did not seem to understand how clicking worked, while another indicated that it was confusing.

With the senior users, it was apparent that some also struggled to operate the mouse. They often double-clicked in a location, causing them to move twice as far away as they wanted. Also, one senior struggled to drag the zoom slider and the map. Slow map response exacerbated these usability problems.

While trying to modify the zoom level, many users were observed clicking on the labels 'Zoom In' and 'Zoom Out', clicking next to the slider, or clicking on the slider track. Also, at least one user attempted to use the zoom control to change the view as you would with a scrollbar. This is an understandable mistake, because the slider is conveniently located where a scrollbar would be.

All of the eighth graders struggled to find their homes. They knew the general area where they lived, but it took some time for them to find it, inquiring about different buildings and roads on the map during the process. This suggests that younger, less-experienced users need additional cues in the form of labels and landmarks.

Interestingly, two of the eighth graders did not recognize the Drillfield (a prominent grassy lawn in the shape of an oval on the Virginia Tech campus) and asked if it was a golf course. All of the seniors and college students easily pointed out this landmark and used it to orient themselves when working with the paper mockups and the map prototype.

\section{Section 3: Paper prototypes of fisheye-view maps}

As the users pointed out places with the different fisheye view paper mockups, we noticed that all of the users preferred the views that contained recognizable landmarks. We expected the users to comment about the advantages and disadvantages of the different projections, but instead they mentioned how important finding a landmark was to complete their task.

We also noticed that if the users recognized a landmark on the map and they pointed out the wrong place, then they were usually close to the target. For example, some of the users pointed out buildings adjacent to the requested post office. The post office is in the heart of the downtown area with many buildings around it and it can be difficult to pick out. This indicates that more visual cues need to be provided to guide the user in the final stages of navigation.

The seniors had many initial comments about fisheye views. These included: 'I'm buffaloed', 'This is really distorted', and 'Main Street is too elongated'. None of the users gave up in looking for the requested places, but these comments expressed their reservations about the fisheye views. 
Also, three of the four eighth graders could not point out a major intersection, not because of problems with the tool, but because they were not familiar with the name of the intersection.

\section{Section 4: Navigation tasks involving fisheye views}

During section three, most of the seniors seemed to forget how to use the map tool. When given a navigation task in section four, they were unsure of how to move the map and had to relearn the interaction techniques. Also, two of the seniors also had to be prompted to zoom out in order to determine where they were located.

Problems with clicking were apparent in this section of the evaluation as well. At least one senior continued struggled with the clicking interaction until the last task. Also, eighth graders were observed accidentally double clicking. This indicates that problems with this interaction technique are not limited to first-time use.

When comparing a fisheye view to the flat view with zoom capabilities, three out of four of the college students found the requested location faster with the flat view, where the largest time difference was 11 seconds. All of the seniors and all of the eighth graders completed the task faster with the flat view. Each of the eighth graders took about 25 seconds longer with the fisheye view, while two of the seniors really struggled with the distorted views. They took 100 to 200 seconds more to complete the fisheye task. As a result, figure 4 shows a larger time difference in the average results for the seniors.

\section{Insert figure 4 here}

When comparing a fisheye view to the flat view without zoom capabilities, three out of four of the college students found the requested location faster with the fisheye view (avg. $16.75 \mathrm{sec}$ ) than with the flat view (avg. $54 \mathrm{sec}$ ). Likewise, three out of the four eighth graders completed the task faster with a fisheye view (avg. $32.25 \mathrm{sec}$ ), than the flat view (avg. $71.25 \mathrm{sec}$ ). Lastly, three out of the four seniors found the requested location faster with the fisheye view. The one exception involved an extreme difference of 180 seconds, causing the group's time difference to reflect faster times with the flat view. On average, the other seniors were actually 47 seconds faster with the fisheye view.

\section{Insert figure 5 here}

These results suggest that when zooming is used, flat maps are better than fisheye views. And, when zooming is not used, fisheye views are superior for most users. The seniors took more time to complete the tasks with zooming than the other 
users, suggesting that they struggled with multiple interactions. Also, the average times for the tasks with zooming are less than the average times in the other tasks for two user groups. This suggests that zoomable maps are more efficient for the eighth graders and college students in many tasks.

\section{Strategies}

All of the users became more familiar with the map of Blacksburg as the session progressed. In the last couple of tasks, users could clearly identify the major landmarks they were using and in most cases the subjects were using them to navigate the prototype very efficiently. For example, at least three of the eighth graders were observed using the same navigation approach: identify Main Street, follow it to the Middle School, and then use the Middle School as a reference point.

Using this approach during the navigation sections, the users repeatedly tried to identify the larger buildings in a view and use these buildings to orient themselves. Without building labels this was a challenge at times. Also, users occasionally had trouble because they identified a large building incorrectly. For example, one of the eighth graders became confused when he believed that a large building was the Middle School when it was not.

Throughout the sessions, users also were observed adding to their set of well-known landmarks. For example, after pointing out the library in one task, users would recognize and use the library as a reference point in later tasks.

Lastly, many of the college students actually became disoriented when trying to orient themselves with the familiar Drillfield. The symmetric shape of the Drillfield causes this confusion, because it is hard to distinguish between two similar sides.

\section{DISCUSSION}

\section{Map modifications}

These results directly indicate some modifications that should be made to increase the usability of the map tool. One simple change is to make the labels, slider track, and area next to the zoom control clickable, and move the zoom control to the left of the map. The map prototype also lacks a labeling scheme. In the sessions, we observed users trying to identify roads and buildings. This activity should be supported either though tool tips or fixed labels on the map.

Currently, the map only has two levels of detail - all roads and, for more zoomed-in views, buildings and roads. The results from this evaluation indicate that when users navigate they use both roads and landmarks. The map should be modified to display these guides at all zoom levels. At the most zoomed-out view, an overview map of major roads and major landmarks along with a labeling scheme will better support users Zooming in, the map should display increasing levels of detail. All 
roads could be added to the overview map followed by all buildings at the most zoomed-in view. In this way, the map will support navigation tasks at all zoom levels.

Lastly, changes need to be made to better support interactions with the map prototype. In particular, a clicking solution must be implemented that makes mouse clicks intuitive and catches erroneous double clicks. Animating the map's movement when a click occurs will provide better feedback to the user. Mouse clicks should also be reserved for selecting actual spaces and landmarks in the MOO. In this way, clicking on a marked space or landmark will cause the map to update, and the user's task focus will change from navigation to visiting the place selected.

\section{Lessons learned}

These results can be generalized into a set of suggestions for using map-based navigation in a multi-user environment. Some of these are especially applicable when the environment represents a real place familiar to users. First, individual users have different perceptions of the same place. In this study, we observed users with different mental representations of Blacksburg. For example, most of the eighth graders did not recognize a major intersection in town when we gave them the road names. They may have been familiar with this intersection, but known it by some other way, such as the intersection with the video store. In this way, the eighth graders had a different perception of the intersection and, in turn, Blacksburg.

Similarly, individual users are familiar with different areas of an environment. In the study, some of the users lived in the southeast section of town and were familiar with various places and roads in that area, while other users had little knowledge of that section of town. Also, when the users were asked to point out the post office and the library, they invariably indicated the post office or library in the part of town with which they were most familiar. In designing a map-based navigation tool, it is important to keep these individual differences, both different perceptions and different familiarities, in mind and support navigation of the entire map space.

The evaluation also revealed that different user groups have knowledge of different landmarks in town. In the first section of the evaluation, most of the college students did not recognize the places thought to be major landmarks in town, while none of the eighth graders or the seniors had trouble with these landmarks. Also, the college students were constantly observed using the Drillfield to complete the tasks and yet two eighth graders inquired about this large oval shape during their sessions. This presents an interesting problem of what landmarks to use with a map-based navigation tool. If we include landmarks more familiar to one user group, they may cause the map to be less usable by the others. If we include landmarks familiar to all groups, the map may become cluttered and less usable for everyone. Ideally, the map should include landmarks common to all groups. This evaluation revealed that grocery stores are a good choice. All ages visit grocery stores and they tend to be 
large buildings often located within noticeable shopping plazas. Designers of map-based navigation need to recognize these differences in landmark knowledge and support all user groups, possibly through a set of common landmarks, or by allowing users to select a customized set of landmarks.

In addition to the differences in landmark knowledge, user groups differ in spatial knowledge and this difference is reflected in how they navigate. In our study, the eighth graders were observed to be more landmark-oriented than the other groups. For example, the eighth graders struggled with road-based tasks, such as pointing out a major intersection in town. Eighth graders do not drive and so they are less familiar with the roads in town than other users. We observed that the landmarks were as useful to 'passengers' as the road names were to the drivers. All of the drivers referred to road names throughout the tasks, while all of the eighth graders relied on the landmark knowledge. For example, during the evaluation one eighth grader mentioned that it was easier to navigate with a zoomed-in, roads and buildings view than a roads only view, even though this limited the area shown on the map. The buildings provided this user a primitive form of landmarks to use while navigating. This difference in spatial knowledge also applies to other grade school children who do not drive or have just recently started driving. A map-based navigation tool must take differences in spatial knowledge into account and support both the use of landmark knowledge and the use of route knowledge.

A more general set of suggestions for supporting navigation can be also identified from the observations. First, recognizable landmarks are key for navigation tasks. When the users were asked to point out places on the paper mockups of the fisheye views, all of the users indicated a preference for the views in which they recognized a landmark. This further emphasizes the need for major landmarks to be included on the map. Similarly, while users navigated with the map prototype, they were observed looking for landmarks they recognized in order to orient themselves with the map. One user even commented: 'If you don't have a Drillfield, then you can't work'. This user was looking for the easily recognizable Drillfield and desired to use it to complete most of the navigation navigation tasks. Not all of the users were successful in orienting with the Drillfield though. Some of the college students struggled with the symmetry of this landmark, thinking the north side was actually the south side and the east side was the west. These users needed more than the landmark, they needed clarification of its orientation. A map-based navigation tool must include landmarks and display them so that they are recognizable and usable. Users are also drawn to large buildings. These buildings are prominent on maps and users will try to use them during navigation. During the evaluation, users would often point out a large building on the screen and ask about it. Again, users were trying to recognize a landmark and orient themselves with it. Designers of a map-based navigation tool need to be aware of the larger buildings in the environment and provide users with ways to identify these buildings. 
As each of the evaluation sessions progressed, it became clear that the users were learning. A uniquely-shaped building, used as the target location in one task, was referenced by users in later tasks. Similarly, major roads pointed out earlier in the evaluation were referenced in later tasks. The users learned about other landmarks while working with the map and they reused this knowledge. This notion of learning should be kept in mind while designing a map-based navigation tool. One possibility for providing better support is to allow map annotations for future reference.

In addition to landmarks, users may also need visual reminders of how to navigate with the map. During the evaluation, none of the users used all three interaction techniques while completing the navigation tasks. In fact, most of the seniors had to be prompted to interact with the map when they were first asked to use it. They also had to relearn how to interact with the map after looking at paper mockups. In MOOsburg, visitable landmarks and spaces are marked on the map and they encourage users to click on them, but navigation controls also need to be apparent. The zoom control provides one type of affordance, but others are needed. A map-based navigation tool must provide visibility and affordances to support navigation interactions, possibly through the use of visible control widgets such as scrollbars (Norman 1990).

\section{CONCLUSIONS AND FUTURE WORK}

Maps provide a good interface for place-based navigation. In MOOs, a map allows a user to browse and visit places while maintaining awareness. Our design of a map-based navigation tool for MOOsburg aims to support users navigating a familiar place.

Map-based navigation could also be used with web pages or in a team-based gaming environment. For example, if a collection of web pages dealt with specific places, a map would provide a good navigation tool. In a game, the map would support simple navigation between places and it would provide awareness information about team members and opponents.

We will continue this work by investigating further support for awareness of other users. We plan to incorporate our results from this evaluation into the prototype and add visual cues for awareness information. We also plan to continue to investigate the usefulness of fisheye views with maps and examine the usability differences between the different fisheye projections. Finally, we will investigate support for end-user programming tasks using a map tool, allowing modifications to the space while navigating within it.

\section{ACKNOWLEDGEMENTS}

The authors wish to thank their subjects for their participation in the study. Other members of the MOOsburg group that were also helpful include Cara Struble, Craig Ganoe, Dan Dunlap, Philip Isenhour, and Dennis Neale. This project was possible through support provided by NSF grant DGE-9553458, the Office of Naval Research, and the Hitachi Foundation. 


\section{REFERENCES}

1.Carroll, J.M., Rosson, M.B., Isenhour, P.L., Ganoe, C.H., Dunlap, D.R., Fogarty, J., Schafer, W.A. and Van Metre, C.A. (2001). Designing Our Town: MOOsburg. Accepted for publication in International Journal of Human-Computer Studies.

2.Bowman, D. Wineman, J., Hodges, L., and Allison, D. The Educational Value of an Information-Rich Virtual Environment. Presence: Teleoperators and Virtual Environments, vol. 8, no. 3, June 1999, pp. 317-331.

3.Darken, R., and Cevik, H. Map Usage in Virtual Environments: Orientation Issues. Proceedings of Virtual Reality '99. 1999. IEEE. pp. 133-140.

4.Darken, R.P., and Sibert, J.L. Wayfinding Strategies and Behaviors in Large Virtual Worlds. Proceedings of Human Factors in Computing Systems. 1996. ACM. pp. 142-149.

5.Furnas, G. The fisheye view: A new look at structured files. Technical Memorandum 82-11221-22, Bell Laboratories, 1982.

6.Lamping, J., Rao, R., and Pirolli, P. A focus+context technique based on hyperbolic geometry for visualizing large hierarchies. Proceedings of Human factors in Computing Systems. 1995. ACM. pp. 401-408.

7.Lynch, K. The Image of the City. 1960, Cambridge: MIT Press.

8.Norman, D. The Design of Everyday Things. 1990, New York: Doubleday.

9.Sarkar, M., and Brown, M.H. Graphical Fisheye Views of Graphs. Proceedings of Human Factors in Computing Systems. 1992. ACM. pp. 83-91.

10.Thorndyke, P., and Hayes-Roth, B. Differences in spatial knowledge obtained from maps and navigation. Cognitive $\begin{array}{lllll}\text { Psychology, } & 1982 . & 14: & \text { pp. }\end{array}$ 


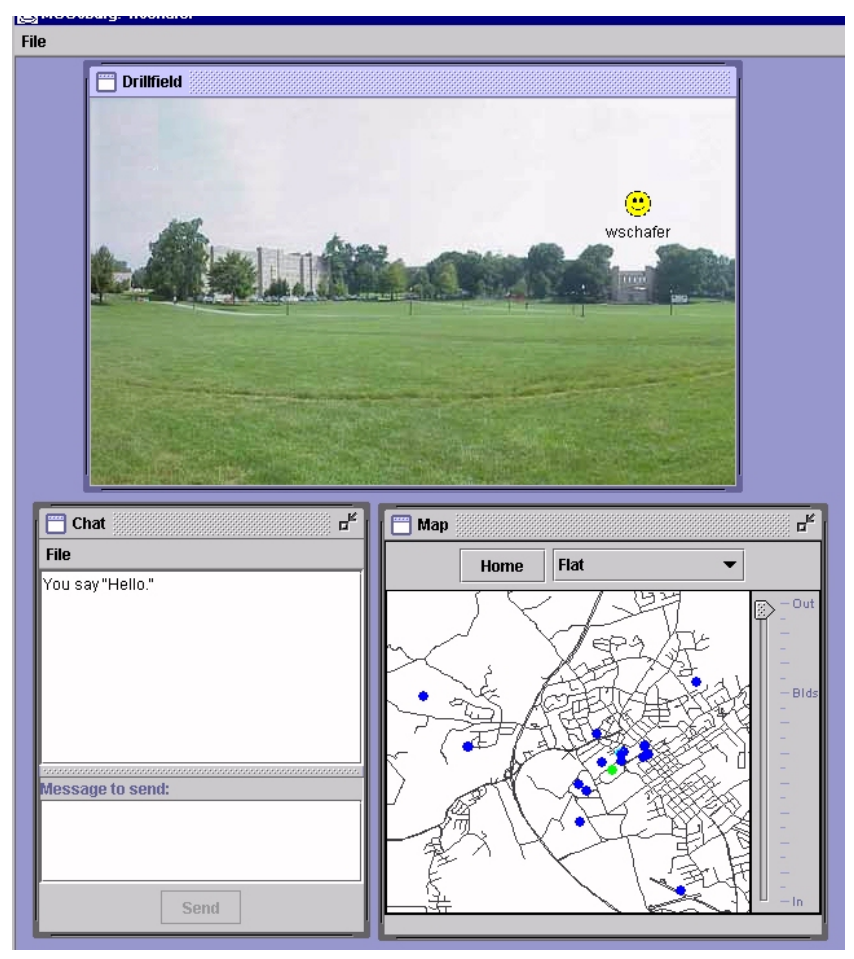

Figure 1. MOOsburg interface, including the map tool (lower right). User 'wschafer' is visiting the Drillfield landmark.
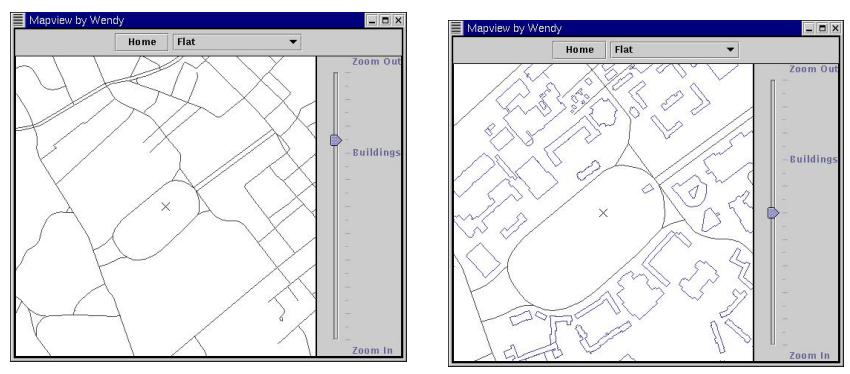

Figure 2. The effect of zooming with the map prototype in the flat view.
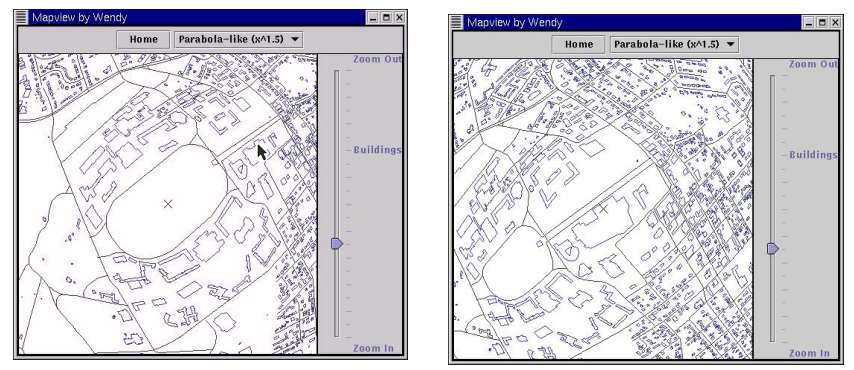

Figure 3. The effect of clicking with the map prototype in the Parabolic $x^{2}$ view. 


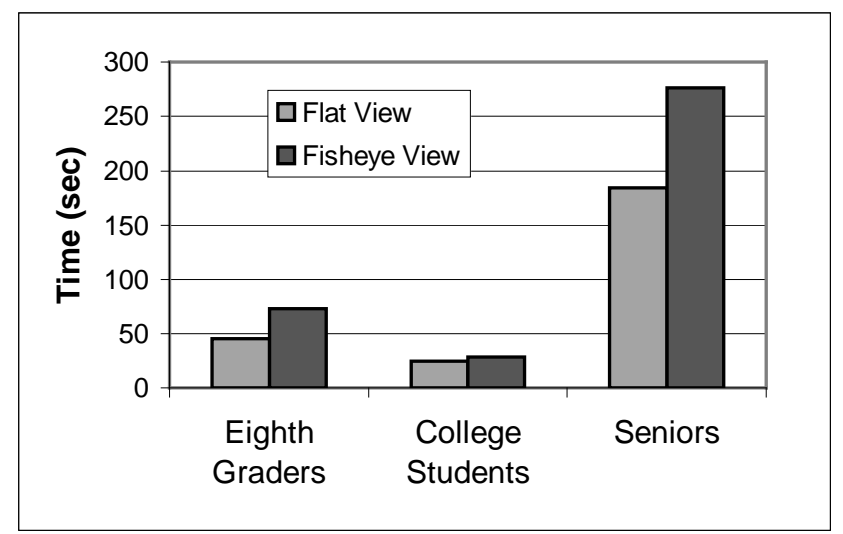

Figure 4. Average navigation times using zooming, by user group and map type.

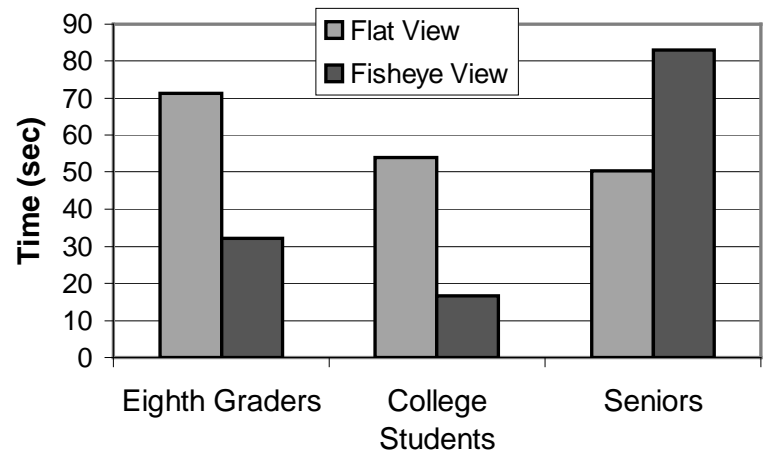

Figure 5. Average navigation times without zooming, by user group and map type.

11. 